Proof. Implication (iii) $\Rightarrow$ (i) is an immediate consequence of Theorem 2.4 because if $\pi$ is strong then $\pi$ is a normal property (cf. [5] Theorem 1, or [9] Theorem 1).

We shall prove (i) $\Rightarrow$ (ii). If $R$ is a ring with identity and $e=e^{2} \epsilon R$ then $R e$ is a projective $R$-module with the representation $(R, e)$ and Hom $(R e, R e)=e R e$. Thus

$$
\begin{aligned}
K(e R e)=K(\operatorname{Hom}(R e, R e)) \subseteq \operatorname{Hom}(R e, K(R) R e) & \\
& \subseteq \operatorname{Hom}(R, K(R) R)=K(R) .
\end{aligned}
$$

To prove (ii) $\Rightarrow$ (iii) let us consider a nil-ring $A$ and let us put $R=A^{\#}$. Of course $A=K(R)$. We write $e=\left(\begin{array}{ll}1 & 0 \\ 0 & 0\end{array}\right) \in R_{2}$. It is easy to see that $e=e^{2}$ and that a ring $e A_{2} e$ isomorphic with $A$ is a $\hbar$-radical of a ring $e R_{2} e$ isomorphic with $R$. Hence

$$
A_{2}=R_{2} e A_{2} e R_{2}=R_{2} \cdot K\left(e R_{2} e\right) \cdot R_{2} \subseteq R_{2} K\left(R_{2}\right) R_{2} \subseteq K\left(R_{2}\right) .
$$

This means that a matrix ring $A_{2}$ is nil for every nil ring $A$. In this case the problem of Koethe has a positive solution, as was proved by J. Krempa [7] and A. D. Sands [9].

\title{
References
}

[1] S. A. Amitsur, A general theory of radicals III, Amer. J. Math. 76 (1954), pp. 126-136.

[2] - Rings of quotients and Morita contexts, J. Algebra 17 (1971), pp. 273-298.

[3] T. Anderson, N. Divinsky and A. Suliński, Hereditary radicals in associative and alternative rings, Canad. J. Math. 17 (1965), pp. 594-603.

[4] N. Divinsky, Rings and radicals, University of Toronto Press, Toronto 1965.

[5] M. Jaegermann, Morita contexts and radicals, Bull. Acad. Polon. Sci. 20 (1972), pp. 619-623.

[6] G. Koethe, Die Struktur der Ringe deren Restklassenring nach dem Radical vollstaendig reduzibel ist, Math. Zeit. 32 (1930), pp. 161-186.

[7] J. Krempa, Logical connections among open problems on noncommutative rings, Fund. Math. 76 (1972), pp. 121-130.

[8] E. M. Patterson, On the radicals of certain rings of infinite matrices, Proc. Royal Soc. Edinburgh 65 (1961), pp. 263-271.

[9] A. D. Sands, Radicals and Morita contexts, J. Algebra 24 (1973), pp. 335-345.

[10] N. E. Sexauer and J. E. Warnock, The radical of the row-finite matrices over an arbitrary ring, Trans. Amer. Math. Soc. 139 (1969), pp. 287-295.

[11] R. Ware and J. Zelmanowitz, The Jacobson radical of the endomorphi $\mathrm{sm}$ ring of a projective module, Proc. Amer. Math. Soc. 26 (1970), pp. 15-20.

Reçu par la Rédaction le 6. 5. 1973

\section{On shapes of topological spaces}

by

Kiiti Morita (Tokyo)

Abstract. A new approach to shapes of topological spaces and its applications will be given.

The notion of shape was originally introduced by K. Borsuk [1] for the case of compact metric spaces. Since then, this notion has been extended to the case of compact Hausdorff spaces by S. Mardešic and J. Segal [12] (cf. also W. Holsztyński [7]) and to the case of metric spaces by K. Borsuk [2] and R. H. Fox [4]. More recently the notion has been extended to the case of arbitrary topological spaces by S. Mardešic [11].

In this note we shall discuss shapes of topological spaces in the sense of Mardešic from another point of view.

For any. category $\mathfrak{C}$, let us denote by Ob $\mathfrak{C}$ the class of all objects of $\mathfrak{E}$, and by $f \in \mathbb{E}(X, Y)$ we mean that $f$ is a morphism from $X$ to $Y$ in $\mathfrak{C}$.

1. Let $\mathfrak{S}$ be the homotopy category of topological spaces. Its objects are topological spaces and its morphisms are homotopy classes of continuous maps; the homotopy class of a continuous map $f: X \rightarrow Y$ will be denoted as usual by $[f]$. Let $\mathfrak{M}$ be the full subcategory of $\mathfrak{S}$ whose objects are all topological spaces having the homotopy type of a CW complex. Throughout this paper, by an ANR we shall mean an ANR for the class of metrizable spaces. The following result is known (cf. Mardešić [11]).

LEMMA 1.1. For a space $X$ the following conditions are equivalent.

(a) $X$ has the homotopy type of a $\mathrm{CW}$ complex.

(b) $X$ has the homotopy type of a simplicial complex with the weak topology (or with the metric topology).

(c) $X$ has the homotopy type of an ANR.

DEFINITION 1.2. Let $\left\{X_{a},\left[p_{a a^{\prime}}\right], A\right\}$ be an inverse system in the category $\mathfrak{S}$ or $\mathfrak{W}$; that is, $A$ is a directed set, continuous maps $p_{\alpha \alpha^{\prime}}: X_{a^{\prime}} \rightarrow X$ $a$ are defined for any $\alpha, \alpha^{\prime}$ with $\alpha<\alpha^{\prime}$, and $\left[p_{\alpha \alpha^{\prime}}\right]\left[p_{\alpha^{\prime} \alpha^{\prime \prime}}\right]=\left[p_{\alpha \alpha^{\prime \prime}}\right]$ if $\alpha<\alpha^{\prime}<\alpha^{\prime \prime}$. We shall say that an inverse system $\left\{X_{\alpha},\left[p_{a \alpha^{\prime}}\right], \mathcal{A}\right\}$ in $\mathfrak{S}$ or 
$\mathfrak{B}$ is associated with a topological space $X$ if there are continuous maps $p_{a}: X \rightarrow X_{a}$ for $\alpha \in A$ such that the following conditions are satisfied.

(i) $\left[p_{\alpha \alpha^{\prime}}\right]\left[p_{\alpha^{\prime}}\right]=\left[p_{\alpha}\right]$, if $\alpha<\alpha^{\prime}$.

(ii) For any continuous map $f: X \rightarrow Q$ with $Q \in \mathrm{Ob}(\mathfrak{W})$ there exist $\alpha \in A$ and a continuous map $f_{a}: X_{a} \rightarrow Q$ such that $[f]=\left[f_{a}\right]\left[p_{a}\right]$.

(iii) For $\alpha \in A$ and for two continuous maps $f_{a}, g_{a}: X_{\alpha} \rightarrow Q$ with $Q \in \mathrm{Ob}(\mathfrak{W})$ such that $\left[f_{a}\right]\left[p_{a}\right]=\left[g_{\alpha}\right]\left[p_{\alpha}\right]$, there exists $\alpha^{\prime} \in A$ with $\alpha<\alpha^{\prime}$ such that $\left[f_{a}\right]\left[p_{a \alpha^{\prime}}\right]=\left[g_{a}\right]\left[p_{a a^{\prime}}\right]$.

For any topological space $X$ there exists an inverse system in $\mathfrak{W}$ associated with $X$.

To prove this, let $\left\{\mathrm{U}_{\alpha} \mid \alpha \in A\right\}$ be the set of all locally finite normal open coverings of $X$. If ${\mathcal{\alpha ^ { \prime }}}$ is a refinement of $\mathcal{U}_{\alpha}$ we write $\alpha<\alpha^{\prime}$. Let $K_{a}$ be the nerve of $\mathcal{U}_{\alpha}$ which we consider a simplicial complex with the weak topology. Then for each $\alpha$ there is a continuous map $p_{\alpha}: X \rightarrow K_{\alpha}$ such that

$$
p_{\alpha}^{-1}\left(\operatorname{St}\left(u ; K_{\alpha}\right)\right) \subset U
$$

where $u$ is a vertex of $K_{a}$ corresponding to the set $U$ of the covering $u_{a}$; such a map $p_{\alpha}$ is called a canonical map and any two canonical maps are homotopic to one another. If $\alpha<\alpha^{\prime}$ we have a simplicial map $p_{\alpha \alpha^{\prime}}: K_{\alpha^{\prime}} \rightarrow K_{a}$ such that $p_{\alpha \alpha^{\prime}}(u)=v$ implies $U \subset V$ where $u$ and $v$ are vertices of $K_{\alpha^{\prime}}$ and $K_{a}$ which correspond to the members $U$ and $\nabla$ of $\varkappa_{a^{\prime}}$ and $\mathcal{U}_{a}$ respectively. Such a map $p_{\alpha \alpha^{\prime}}$ is called a canonical projection and any two canonical projections are homotopic. Moreover, we have

$$
\left[p_{\alpha \alpha^{\prime}}\right]\left[p_{\alpha^{\prime}}\right]=\left[p_{\alpha}\right] \quad \text { if } \quad \alpha<\alpha^{\prime} .
$$

Thus, we obtain the following theorem from Morita [16, Theorem 4.3].

THEOREM 1.3. With the above notations, $\left\{K_{\alpha},\left[p_{a a^{\prime}}\right], A\right\}$ is an inverse system in $\mathfrak{W}$ associated with the space $X$.

In case $X$ is metrizable we have another inverse system in $\mathfrak{W}$ associated with $X$.

For any metrizable space $X$, by a well-known theorem of Kuratowski-Wojdysławski there exists a metrizable space $P$ which is an ANR and contains $X$ as a closed subset. More generally, let $P$ be any ANR such that $X \subset P$. Then the set $\{G\}$ of all open neighborhoods $G$ of $X$ in $P$ forms an inverse system $\left\{G, i_{G G^{\prime}}\right\}$ of ANR's with inclusion maps $i_{G G^{\prime}}$ as bonding maps, which will be denoted by $U(X, P)$ and is called the complete neighborhood system of $X$ in $P$ (cf. Fox [4]). In this case $\left\{G,\left[i_{G G^{\prime}}\right]\right\}$ is
an inverse system in $\mathfrak{W}$.

THEOREM 1.4. The complete neighborhood system $U(X, P)$ of $X$ in $P$ induces an inverse system in $\mathfrak{W}$ associated with $X$.
For compact Hausdorff spaces we have the following two theorems; the first is known and the second is due to Mardesice [11].

THEOREM 1.5. For any compact Hausdorff space $X$ there is an inverse system of finite polyhedra which has $X$ as its inverse limit.

THEOREM 1.6. Let $\left\{X_{a}, p_{a a^{\prime}}, A\right\}$ be an inverse system of compact Hausdorff spaces with $X$ as its inverse limit. Then the inverse system $\left\{X_{\alpha},\left[p_{\alpha \alpha^{\prime}}\right], A\right\}$ in $\mathfrak{S}$ is associated with $X$.

To obtain Theorems 1.4 and 1.6 simultaneously, we shall give the following definition.

DEFINITION 1.7. Let $\left\{X_{a}, p_{\alpha \alpha^{\prime}}, A\right\}$ be an inverse system of topological spaces with $X$ as its inverse limit; let $p_{a}: X \rightarrow X_{a}, \alpha \in A$, be the projections. We shall say that a covering $\mho$ of $X_{a}$ is proper (with respect to $p_{a}$ ) if $\mathcal{V}$ is a locally finite normal open covering such that the nerve of $\mathcal{V}$ is isomorphic to the nerve of $p_{a}^{-1}(\mathcal{V})$ under the map $p_{a}^{-1}$. In case for any $\beta \in A$ and for any locally finite normal open coverings $\mathcal{G}$ of $X$ and Je of $X_{\beta}$ there exist $\alpha \in A$ with $\beta<\alpha$ and a proper covering $\vartheta$ of $X_{\alpha}$ such that $p_{\alpha}^{-1}(\mathcal{\cup})$ refines $\mathcal{S}$ and $\mathcal{V}$ refines $p_{\beta a}^{-1}(\mathcal{H})$, we call the inverse system $\left\{X_{a}, p_{a \alpha^{\prime}}, A\right\}$ proper $\left(^{1}\right)$.

LEMMA 1.8. The inverse systems described in Theorems 1.4 and 1.6 are proper.

Proof. Let $P$ be a metric space with a metric $\varrho$ containing $X$. To each open subset $G$ of $X$ we can correspond an open subset $\psi(G)$ of $P$ such that $\psi(G) \cap X=G$ and such that $\bigcap_{i=1}^{m} G_{i}=\varnothing$ implies $\bigcap_{i=1}^{m} \psi\left(G_{i}\right)=\varnothing$; indeed, one defines $\psi(G)=\{p \in P \mid \varrho(p, G)<\varrho(p, X-G)\}$. This fact is due to Kuratowski [10, p. 122].

Let $V$ be an open neighborhood of $X$, and let $\mathcal{G}$ (resp. He) be a locally finite normal open covering of $X$ (resp. $V$ ). Let $\left\{G_{\lambda} \mid \lambda \in \Lambda\right\}$ be any locally

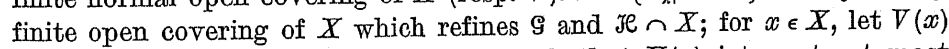
be an open neighborhood of $x$ in $X$ such that $V(x)$ intersects at most finitely many sets $G_{\lambda}$. Let us put $\psi_{0}\left(G_{\lambda}\right)=\psi\left(G_{\lambda}\right) \cap H_{\alpha(\lambda)}$ and

$$
U_{0}=\bigcup\{\psi(V(x)) \mid x \in X\}, \quad U=\bigcup\left\{\psi_{0}\left(G_{\lambda}\right) \cap U_{0} \mid \lambda \in \Lambda\right\},
$$

where we assume $G_{\lambda} \subset H_{a(\lambda)}, H_{a(\lambda)} \in$ Je. Then $X \subset U \subset \nabla, U$ is open in $P$ and $\left\{\psi_{0}\left(G_{\lambda}\right) \cap U_{0} \mid \lambda \in \Lambda\right\}$ is a proper covering of $U$ with respect to the inclusion $\operatorname{map} X \subset U$.

Next, let $\left\{X_{a}, p_{a \alpha}, A\right\}$ and $X$ be the same as in Theorem 1.6. Let $\mathbf{S}$ and Je be any open coverings of $X$ and of $X_{\beta}$ respectively. Then there are $\alpha \in A$ with $\beta<\alpha$ and a covering $U=\left\{U_{i} \mid i=1, \ldots, m\right\}$ of $X_{\alpha}$ by open

(1) I am indebted to Philip Bacon for calling my attention to a defect of the definition of "proper" in the original version of the paper. 
$F_{\sigma}$-subsets such that $น$ refines $p_{\beta \alpha}^{-1}(\mathcal{H})$ and $p_{\alpha}^{-1}(\mathcal{U})$ refines 9. By $[14$, Lemma 1] there are open $F_{\sigma}$-subsets $\nabla_{i}, i=1, \ldots, m$, of $X_{a}$ such that $J_{i} \cap p_{a}(X)=V_{i} \cap p_{a}(X), \nabla_{i} \subset V_{i}$ for each $i$ and such that $\left\{V_{i}\right\}$ is similar to $\left\{U_{i} \cap p_{a}(X)\right\}$. As is known, there is $\alpha^{\prime} \in A$ with $\alpha<\alpha^{\prime}$ such that $p_{a a^{\prime}}\left(X_{n^{\prime}}\right)$ $C \bigcup^{\prime} \nabla_{i}$. Thus $V=\left\{p_{a a^{\prime}}^{-1}\left(V_{i}\right)\right\}$, is a proper covering of $X_{a^{\prime}}$ which refines $p_{\beta \alpha^{\prime}}^{-1}(\mathcal{H})$, and $p_{\alpha^{\prime}}^{-1}(\mathcal{V})$ refines $\mathcal{G}$. This completes the proof of Lemma 1.8 .

In view of Lemma 1.8, Theorems 1.4 and 1.6 are direct consequences of Theorem 1.9 below.

THEOREM 1.9. Let $\left\{X_{\alpha}, p_{\alpha \alpha^{\prime}}, A\right\}$ be a proper inverse system of topological spaces with $X$ as its inverse limit. Then the inverse system $\left\{X_{a},\left[p_{a a^{\prime}}\right], A\right\}$ in $\mathfrak{S}$ is associated with $X$.

Proof. Let $N$ denote the operation of talking the nerve of a covering.

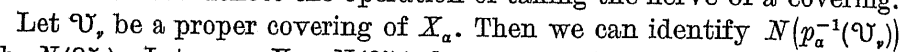
with $N\left(\mathcal{V}_{v}\right)$. Let $\varphi_{y a}: X_{\alpha} \rightarrow N\left(\mathcal{V}_{v}\right)$ be a canonical map. Then $\varphi_{v a} p_{a}$ : $X \rightarrow N\left(p_{a}^{-1}\left(V_{,}\right)\right)$is a canonical map.

Let $f: X \rightarrow Q$ be a continuous map with $Q \in \mathrm{Ob}(\mathfrak{W})$. Then by Theorem 1.3 and the assumption of Theorem 1.9 there exist $\alpha \in A$, a proper covering $\mathcal{V}_{\nu}$ of $X_{a}$, and a continuous map $f_{v}^{\prime}: N\left(p_{a}^{-1}\left(\mho_{v}\right)\right) \rightarrow Q$ such that $[f]=\left[f_{v}^{\prime}\right]\left[\varphi_{v}\right]$, where $\varphi_{v}: X \rightarrow N\left(p_{a}^{-1}\left(\mathcal{V}_{v}\right)\right)$ is a canonical map. Since $\left[\varphi_{v}\right]$ $=\left[p_{v a}\right]\left[p_{a}\right]$, we have $[f]=\left[f_{a}\right]\left[p_{a}\right]$ by putting $f_{\alpha}=f_{\nu}^{\prime} p_{v \alpha}: X_{\alpha} \rightarrow Q$.

Next, let $\alpha<\alpha^{\prime}$ and let $\mathcal{U}_{\lambda}$ be a proper covering of $X_{\alpha^{\prime}}$ which refines

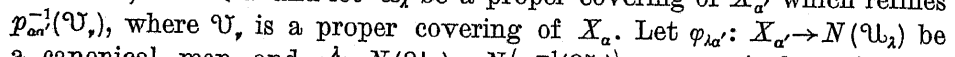
a canonical map and $\varphi_{\nu}^{\lambda}: N\left(\mathcal{U}_{\lambda}\right) \rightarrow N\left(p_{a a^{\prime}}^{-1}\left(\mho_{\nu}\right)\right)$ a canonical projection. Then $\varphi_{v a} p_{a a^{\prime}}$ and $\varphi_{v}^{\lambda} \varphi_{\lambda a^{\prime}}$ are both canonical maps from $X_{\alpha^{\prime}}$ to $N\left(p_{a a^{\prime}}^{-1}\left(\mho_{v}\right)\right)$. Hence we have

$$
\left[\varphi_{p a}\right]\left[p_{a a^{\prime}}\right]=\left[\varphi_{v}^{\lambda}\right]\left[\varphi_{\lambda \alpha^{\prime}}\right] .
$$

Suppose that $f_{\beta}, g_{\beta}: X_{\beta} \rightarrow Q$ are continuous maps with $\beta \in A,\left[f_{\beta}\right]\left[p_{\beta}\right]$ $=\left[g_{\beta}\right]\left[p_{\beta}\right]$ and that $Q$ is a simplicial complex with the weals topology. Let us put $\mathscr{H}=\{\operatorname{St}(q ; Q) \mid q$ ranging over all vertices of $Q\}$. Then there exist $\alpha \in A$ with $\beta<\alpha$ and a proper covering $V_{v}$ of $X_{\alpha}$ such that $p_{\alpha}^{-1}\left(V_{v}\right)$ refines $\left(f_{B} p_{\beta}\right)^{-1}(\mathcal{M})$ and $\left(g_{\beta} p_{\beta}\right)^{-1}(\mathcal{M})$, and $\mathcal{V}_{\text {, refines }}\left(f_{\beta} p_{\beta a}\right)^{-1}(\mathcal{M})$ and $\left(g_{\beta} p_{\beta \alpha}\right)^{-1}(\mathcal{M})$. Hence by [16, Lemma 4.1] there exist continuous maps $f_{v}^{\prime}, g_{v}^{\prime}: N\left(\mho_{v}\right) \rightarrow Q$
such that

$$
\left[f_{\beta} p_{\beta a}\right]=\left[f_{r}^{\prime}\right]\left[\varphi_{v a}\right], \quad\left[g_{\beta} p_{\beta a}\right]=\left[g_{v}^{\prime}\right]\left[\varphi_{v a}\right] .
$$

Since $\varphi_{v a} p_{a}: X \rightarrow N\left(\mho_{v}\right)=N\left(p_{a}^{-1}\left(\mho_{v}\right)\right)$ is a canonical map, we have $\left[f_{v}^{\prime}\right]\left[\varphi_{v}\right]$ $=\left[g_{v}^{\prime}\right]\left[\varphi_{\nu}\right]$ for any canonical map $\varphi_{\nu}: X \rightarrow N\left(p_{a}^{-1}\left(\vartheta_{v}\right)\right)$.

Therefore, by Theorem 1.3 there exist $\alpha^{\prime} \in A$ with $\alpha<\alpha^{\prime}$ and a proper covering $\mathcal{U}_{\lambda}$ of $X_{\alpha^{\prime}}$ such that $\mathcal{U}_{\lambda}$ refines $p_{a \alpha^{\prime}}^{-1}\left(\mathcal{V}_{v}\right)$ and

$$
\left[f_{v}^{\prime}\right]\left[\varphi_{v}^{\lambda}\right]=\left[g_{v}^{\prime}\right]\left[\varphi_{v}^{\lambda}\right]
$$

where $\varphi_{\nu}^{\lambda}: N\left(p_{\alpha^{\prime}}^{-1}\left(\mathcal{U}_{\lambda}\right)\right) \rightarrow N\left(p_{\alpha}^{-1}\left(\mathcal{V}_{\nu}\right)\right)$ is a canonical projection. We note that $\varphi_{p}^{\lambda}$ may be identified with $\varphi_{v}^{\lambda}$ defined before since $U_{\lambda}$ is a proper covering refining $p_{a a^{\prime}}^{-1}\left(\mho_{p}\right)$. Hence, by (1), (2) and (3) we have

$$
\left[f_{\beta}\right]\left[p_{\beta a^{\prime}}\right]=\left[g_{\beta}\right]\left[p_{\beta a^{\prime}}\right] \text {. }
$$

This completes the proof of Theorem 1.9.

2. Following Mardešic [11] we shall define the shape category $\mathfrak{S}$ of topological spaces as follows. The objects of $\mathbb{S}$ are topological spaces. Let $X$ and $Y$ be topological spaces. The morphisms of $\subseteq$ are called shapings (or shape maps). A shaping $f: X \rightarrow Y$ is a function which assigns to every $\eta \in \mathfrak{S}(\bar{Y}, Q)$ with $Q \in \mathrm{Ob}(\mathfrak{M})$ a homotopy class $f(\eta) \in \mathfrak{S}(X, Q)$ in such a way that for $Q^{\prime} \in \mathrm{Ob}(\mathfrak{W}), \eta^{\prime} \in \mathfrak{S}\left(\bar{Y}, Q^{\prime}\right)$ and $\mu \in \mathfrak{W}\left(Q, Q^{\prime}\right)$ the equality $\mu \eta=\eta^{\prime}$ implies $\mu f(\eta)=f\left(\eta^{\prime}\right)$.

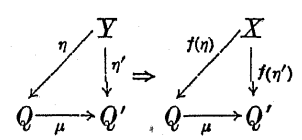

If $X, Y, Z$ are topological spaces and $f: X \rightarrow Y, g: Y \rightarrow Z$ are shapings, then the composite $g f: X \rightarrow Z$ is defined by $(g f)(\zeta)=f(g(\zeta))$ for $\zeta \in \mathfrak{H}(Z, Q)$ with $Q \in \mathrm{Ob}(\mathfrak{M})$. The identity shaping $1_{X}: X \rightarrow X$ is defined by $1_{X}(\xi)=\xi$ for $\xi \in \mathfrak{H}(X, Q)$ with $Q \in \mathrm{Ob}(\mathfrak{B})$.

Mardesic [11] proved that $\mathbb{S}$ is actually a category.

The shape functor $S: \mathfrak{S} \rightarrow \mathbb{S}$ is defined as follows: $S(X)=X$ for every space $X$, and if $\varphi: X \rightarrow Y$ is a homotopy class of continuous maps, the shaping $S(\varphi): X \rightarrow Y$ is defined by $S(\varphi)(\eta)=\eta \varphi$ for $\eta \in \mathfrak{T}(Y, Q)$ with $Q \in \mathrm{Ob}(\mathfrak{W}) . S$ is a covariant functor. Mardešic [11] proved the following theorems.

THEOREM 2.1. Let $Y \in \mathrm{Ob}(\mathfrak{M})$. If $f: X \rightarrow Y$ is a shaping then there is a unique homotopy class $\varphi: X \rightarrow Y$ such that $S(\varphi)=f$.

THEOREM 2.2. If $f: X \rightarrow Y$ is a shaping and $\eta \in \mathfrak{S}(\bar{Y}, Q), Q \in \mathrm{Ob}(\mathfrak{M})$ then $S(\eta) f=S(f(\eta))$.

We shall now state the following theorem.

THEOREM 2.3. Let $X$ be a topological space and $\left\{Y_{\beta},\left[q_{\beta \beta^{\prime}}\right], B\right\}$ an inverse system in the category $\mathfrak{W}$ which is associated with another topological space $Y$; let $q_{\beta}: X \rightarrow Y_{\beta}, \beta \in B$, be continuous maps satisfying conditions (i) to (iii) in Definition 1.2. Then there is a one-to-one correspondence between shapings $f: X \rightarrow \bar{X}$ and systems of homotopy classes $\varphi_{\beta} \in \mathfrak{H}\left(X, Y_{\beta}\right), \beta \in B$, satisfying

$$
\left[q_{\beta \beta^{\prime}}\right] \varphi_{\beta^{\prime}}=\varphi_{\beta} \quad \text { for } \quad \beta, \beta^{\prime} \text { with } \beta<\beta^{\prime} .
$$

The correspondence is given by $S\left[q_{\beta}\right] f=S\left(\varphi_{\beta}\right), \beta \in B$. 
Proof. In [11, Theorem 7] Mardešić has proved this theorem for the case where $\left\{Y_{\beta}, q_{\beta \beta^{\prime}}, B\right\}$ is an inverse system of compact Hausdorff spaces $\bar{Y}_{\beta} \in \mathrm{Ob}(\mathfrak{B})$ and $\bar{Y}=\lim \left\{\bar{Y}_{\beta}, q_{\beta \beta^{\prime}}, B\right\}$. His proof there is based upon Theorems 2.1, 2.2 and the fact described in Theorem 1.6. Hence the argument in his proof is applicable to the present case and we have Theorem 2.3.

Next, let $\left\{X_{\alpha},\left[p_{a a^{\prime}}\right], A\right\}$ and $\left\{Y_{\beta},\left[q_{\beta \beta^{\prime}}\right], B\right]$ be inverse systems in the category $\mathfrak{W}$. A system map from $\left\{X_{a},\left[p_{a \alpha^{\prime}}\right], A\right\}$ to $\left\{Y_{\beta},\left[q_{\beta \beta^{\prime}}\right], B\right\}$, by definition, consists of a map $\varphi: B \rightarrow A$ and homotopy classes of maps $f_{\beta}: X_{\varphi(\beta)} \rightarrow Y_{\beta}$ for $\beta \in B$ such that, if $\beta<\beta^{\prime}$ in $B$ then

$$
\left[q_{\beta \beta^{\prime}}\right] f_{\beta^{\prime}}\left[p_{\varphi\left(\beta^{\prime}\right) a}\right]=f_{\beta}\left[p_{\varphi(\beta) a}\right]
$$

for some $\alpha$ with $\varphi(\beta)<\alpha$ and $\varphi\left(\beta^{\prime}\right)<\alpha$. Two system maps $\left\{\varphi, f_{\beta}, B\right\}$ and $\left\{\psi, g_{\beta}, B\right\}$ are said to be equivalent if for each $\beta \in B$ there exists $\alpha \in A$ such that $\varphi(\beta)<\alpha, \psi(\beta)<\alpha$ and

$$
f_{\beta}\left[p_{q(\beta) \alpha}\right]=g_{\beta}\left[p_{\psi(\beta) \alpha}\right] .
$$

Then the argument in Mardesic $[11, \S 7]$ is applicable to the present case by virtue of Theorem 2.3. Hence we have the following theorem.

THEOREM 2.4. Suppose that inverse systems $\left\{X_{\alpha},\left[p_{\alpha^{\prime}}\right], A\right\}$ and $\left\{Y_{\beta},\left[q_{\beta \beta^{\prime}}\right], B\right\}$ in $\mathfrak{M}$ are associated with the spaces $X$ and $Y$ respectively. Then there is a one-to-one correspondence between shapings $f: X \rightarrow Y$ and equivalence classes of system maps from $\left\{X_{a},\left[p_{a a^{\prime}}\right], A\right\}$ to $\left\{Y_{\beta},\left[q_{\beta \beta^{\prime}}\right], B\right\}$.

Of course, to the identity shaping $1_{X}: X \rightarrow X$ there corresponds the equivalence class of the identity map $\left\{1_{A}, 1_{X_{a}}, A\right\}$ from $\left\{X_{\alpha},\left[p_{\alpha \alpha^{\prime}}\right], A\right\}$
to itself. Let

$$
\begin{gathered}
\left\{\varphi, f_{\beta}, B\right\}:\left\{X_{\alpha},\left[p_{\alpha \alpha^{\prime}}\right], A\right\} \rightarrow\left\{Y_{\beta},\left[q_{\beta \beta^{\prime}}\right], B\right\}, \\
\left\{\psi, g_{\gamma}, C\right\}:\left\{Y_{\beta},\left[q_{\beta \beta^{\prime}}\right], B\right\} \rightarrow\left\{Z_{\gamma},\left[r_{\gamma \gamma^{\prime}}\right], C\right\}
\end{gathered}
$$

be system maps in $\mathfrak{W}$. Then the composite of these maps is defined by $\left\{\chi, h_{\gamma}, C\right\}$, where $\chi=\phi \psi: C \rightarrow A$ and $h_{\gamma}=g_{\gamma} f_{\psi(\gamma)}: X_{\chi(\gamma)} \rightarrow Z_{\gamma}$. If $\left\{X_{\alpha},\left[p_{a a^{\prime}}\right], A\right\}$, $\left\{Y_{\beta},\left[q_{\beta \beta^{\prime}}\right], B\right\}$ and $\left\{Z_{\gamma},\left[r_{\gamma \gamma^{\prime}}\right], C\right\}$ are associated with spaces $X, Y$ and $Z$ respectively, then the composite of $\left\{\varphi, f_{\beta}, B\right\}$ and $\left\{\psi, g_{y}, C\right\}$ corresponds to the composite of shapings corresponding to these maps. Therefore by using equivalence classes of system maps of inverse systems in $\mathfrak{W}$ associated with topological spaces we have another approach to the notion of shapings.

By restricting the inverse systems under consideration to ones described in Theorem 1.4 or 1.5 , we have an approach to shapings in the case of metrizable spaces or in the case of compact Hausdorff spaces.
Such an approach is due to Fox [4] for the former case $\left({ }^{2}\right)$ and to Mardešić and. Segal [11] for the latter case.

In case there are shapings $f: X \rightarrow Y$ and $g: Y \rightarrow X$ such that $g f=1_{X}$ and $f g=1_{\bar{X}}, X$ and $Y$ are said to have the same shape and we write $\operatorname{Sh}(X)$ $=\mathrm{Sh}(Y)$. Thus, we have

THEOREM 2.5. The notion of shape in the sense of Mardešic coincides with that in the sense of Fox [4] for the case of metrizable spaces, and with that in the sense of Mardessio and Segal [12] for the case of compact Hausdorff spaces.

The second assertion in Theorem 2.5 is due to Mardešić [11]. It should be noted that for the case of compact metric spaces the notion of shape in the original sense of Borsuk coincides with that in the sense of Fox [4] or in the sense of Mardešić and Segal [13], but that two notions of shape due to Borsuk [2] and Fox [4] for the case of metrizable spaces are different (cf. Godlewski and Nowak [6]).

The following theorem is a direct consequence of Theorem 2.3.

THEOREM 2.6. Let $\left\{X_{a},\left[p_{a \alpha^{\prime}}\right], A\right\}$ be an inverse system in $\mathfrak{W}$ associated with a topological space $X$. Then in the shape category $\mathfrak{S}$ the object $X$ is the inverse limit of the inverse system $\left\{X_{a}, S\left(\left[p_{a \alpha^{\prime}}\right]\right), A\right\}$.

3. For any topological space $X$, an Abelian group $G$, and an integer $n \geqslant 0$, let $H_{n}(X ; G)$ (resp. $\left.H^{n}(X ; G)\right)$ be the $n$th Cech homology (resp. cohomology) group based on locally finite normal open coverings; that is, with the notations in $\S 1$, we have

$$
\begin{aligned}
& H_{n}(X ; G)=\lim _{\leftarrow}\left\{H_{n}\left(K_{a} ; G\right),\left(p_{a \alpha^{\prime}}\right)_{*}, A\right\}, \\
& H^{n}(X ; G)=\lim _{\longrightarrow}\left\{H^{n}\left(K_{a} ; G\right),\left(p_{a \alpha^{\prime}}\right)^{*}, A\right\} .
\end{aligned}
$$

Then Theorem 2.3 gives rise to

THEOREM 3.1. If $f: X \rightarrow Y$ is a shaping, then $f$ induces homomorphisms $f_{*}: H_{n}(X ; G) \rightarrow H_{n}(Y ; G)$ and $f^{*}: H^{n}(Y ; G) \rightarrow H^{n}(X ; G)$. Hence, if $\operatorname{Sh}(X)$ $=\operatorname{Sh}(Y)$, then $H_{n}(X ; G) \cong H_{n}(Y ; G)$ and $H^{n}(X ; G) \cong H^{n}(Y ; G)$.

It is stated in [11] that the last assertion has been obtained by H. Breger.

THEOREM 3.2. Let $\left\{Y_{\beta},\left[q_{\beta \beta^{\prime}}\right], B\right\}$ be an inverse system in the category $\mathfrak{W}$ which is associated with a topological space $X$. Then we have

$$
\begin{aligned}
& \mathbb{H}^{n}(X ; G) \cong \lim _{\longrightarrow}\left\{H^{n}\left(Y_{\beta} ; G\right),\left(q_{\beta \beta^{\prime}}\right)^{*}, B\right\}, \\
& \mathbb{H}_{n}(X ; G) \cong \lim _{\leftarrow}\left\{H_{n}\left(Y_{\beta} ; G\right),\left(q_{\beta \beta^{\prime}}\right)_{*}, B\right\} .
\end{aligned}
$$

(2) For $U(X, P)$ Fox [4] had made the restriction that $X$ be closed in $P$, but it was shown by Hyman [8] that this restriction can be removed. 
Proof. Let $\left\{K_{\alpha},\left[p_{a a^{\prime}}\right], A\right\}$ be the inverse system described in Theorem 1.3. Then by Theorems 1.3 and 2.4 there exist two system maps

$$
\begin{aligned}
& \Phi=\left\{\varphi, f_{\beta}, B\right\}:\left\{K_{a},\left[p_{\alpha \alpha^{\prime}}\right], A\right\} \rightarrow\left\{Y_{\beta},\left[q_{\beta \beta^{\prime}}\right], B\right\}, \\
& \Psi=\left\{\psi, g_{\alpha}, A\right\}:\left\{Y_{\beta},\left[q_{\beta \beta^{\prime}}\right], B\right\} \rightarrow\left\{K_{a},\left[p_{\alpha \alpha^{\prime}}\right], A\right\},
\end{aligned}
$$

such that the composites $\Psi \Phi$ and $\Phi \Psi$ are equivalent to the respective identity maps $\left\{1_{A}, 1_{K_{a}}, A\right\}$ and $\left\{1_{B}, 1_{Y_{\beta}}, B\right\}$. Hence we have the theorem.

Remark. If $\left\{Y_{\beta},\left[q_{\beta \beta^{\prime}}\right], B\right\}$ is an inverse system in the homotopy category $\mathfrak{H}$ and is associated with $X$, then we have also $H^{n}(X ; G) \cong$ $\lim \left\{H^{n}\left(Y_{\beta} ; G\right),\left(q_{\beta \beta^{\circ}}\right)^{*}, B\right\}$ by using [16, Theorem 6.2].

4. In our previous paper [16], the covering dimension of a topological space $X$, denoted by $\operatorname{dim} X$, is defined to be the least integer $n$ such that every finite normal open covering of $X$ is refined by a finite normal open covering of order $\leqslant n+1$; if there is no such an integer $n$, we write $\operatorname{dim} X=\infty$. Let us define the shape dimension of $X$, Sd $X$, to be $\operatorname{Min}\{\operatorname{dim} Y \mid \operatorname{Sh}(X) \leqslant \operatorname{Sh}(Y)\}$. Here $X$ is said to be shape dominated by $\bar{X}$, $\operatorname{Sh}(X) \leqslant \operatorname{Sh}(Y)$ in notation, if there are shapings $f: X \rightarrow Y$ and $g: Y \rightarrow X$ such that $g f=1_{X}$.

Since by [16, Theorem 6.7] every continuous map $f: X \rightarrow S^{n}\left(S^{n}\right.$ being an $n$-sphere) is inessential if $\operatorname{dim} X<n$, we can prove the following theorem similarly as in Godlewski and Holsztyński [5].

THEOREM 4.1. If $\operatorname{Sd} X<n$, then every continuous map $f: X \rightarrow S^{n}$ is inessential.

5. As is described in [16], there exists a reflector $\tau$ from the category of topological spaces and continuous maps to the full subcategory of Tychonoff spaces; that is, $\tau(X)$ is a Tychonoff space, a natural continuous map $\Phi_{X}: X \rightarrow \tau(X)$ is defined, and any continuous map $f: X \rightarrow Q$ with $Q$ a Tychonoff space is factored through $\tau(X)$ such that $f$ $=\Phi_{Q}^{-1} \tau(f) \cdot \Phi_{X}$. Since $\tau(X \times I)=\tau(X) \times I$ by Puppier [17] (cf. also [16, Lemma 1.4]), $f \simeq g: X \rightarrow Q$ implies $\tau(f) \simeq \tau(g): \tau(X) \rightarrow \tau(Q)$, where $I$ is the closed unit interval $[0,1]$.

If we let $Q$ range over all ANR's and assign to any continuous map $f: X \rightarrow Q$ the continuous map $\Phi_{Q}^{-1} \tau(f): \tau(X) \rightarrow Q$, then we have a shaping $\psi: \tau(X) \rightarrow X$. It is obvious that $S\left(\left[\Phi_{X}\right]\right) \psi=1_{\tau(X)}$ and $\psi S\left(\left[\Phi_{X}\right]\right)=1_{X}$. Hence we have

THEOREM 5.1. A topological space $X$ has the same shape as $\tau(X)$.

For a Tychonoff space $X$ let us denote by $\mu(X)$ the completion of $X$ with respect to its finest uniformity. Then any continuous map from $\bar{X}$ into a metric space can be extended over $\mu(X)$. Moreover, $\mu(X \times I)$ $=\mu X \times I$ as was proved by Puppier [17] and Morita [15] independently. Hence we have the following theorem similarly as above.
THEOREM 5.2. If $X$ is a Tychonoff space, then $X$ and $\mu(X)$ are of the same shape.

Since $\mu(X)=\beta(X)$ if and only if $X$ is pseudocompact ([15]), we have the following corollary.

COROLLARY 5.3. A pseudocompact space has the shape of a compact Hausdorff space.

Added in proof. The first assertion of Theorem 2.5 was proved independently by S. Mardešic (Equivalence of two notions of shape for metric spaces, Bull. Acad. Polon. Sci., Sór. Sci. Math. Astronom. et Phys. 21 (1973), pp. 1137-1142).

\section{References}

[1] K. B orsulk, Ooncerning the homotopy properties of compacta, Fund. Math. 62 (1968) pp. '223-254.

[2] - On the concept of shape for metrizable spaces, Bull. Acad. Polon. Sci., Sér. Sci. Math. Astronom. Phys. 18 (1970), pp. 127-132.

[3] - A note on the theory of shape of compacta, Fund. Math. 67 (1970), pp. 265-278.

[4] R. H. Fox, On shape, Fund. Math. 74 (1972), pp. 47-71.

[5] S. Godlewski and W. Holsztyński, Some remarkes concerning Borsuk's theory of shape, Bull. Acad. Polon. Sci. Sér. Sci. Math. Astronom. Phys. 17 (1969), pp. 373-376.

[6] - and S. Nowak, On two notions of shape, Bull. Acad. Polon. Sci. Sér. Sci. Math. Astronom. Phys. 20 (1972), pp. 387-393.

[7] W. Holsztyński, An extension and axiomatic characterization of Borsult's theory of shape, Fund. Math. 70 (1971), pp. 157-168.

[8] D. M. Hymán, A remark on Fox's paper on shape, Fund. Math. 75 (1972), pp. 205-208.

[9] K. Kuperberg, An isomorphism theorem on the Hurewicz-type in Borsuk's theory of shape, Fund. Math. 77 (1972), pp. 21-32.

[10] C. Kuratowski, Topologie I, Warszawa 1948.

[11] S. Mardešić, Shapes for topological spaces (to appear).

[12] S. Mardešić and J. Segal, Shapes of compacta and ANR-systems, Fund. Math. 72 (1971), pp. 41-59.

[13] - - Equivalence of the Borsute and the ANR-system approach to shapes, Fund. Math. 72 (1971), pp. 61-68.

[14] K. Morita, On spaces having the weate topology with respect to closed coverings, II, Proc. Japan Acad. 30 (1954), pp. 711-717.

[15] - Topological completion and M-spaces, Sci. Rep. Tokyo Kyoiku Daigaku, Sect. A, 10 (1970), pp. 271-288.

[16] - Cech cohomology and covering dimension for topological spaces (to appear).

[17] R. Puppier, La completion universelle d'un produit d'espaces completement reguliers, Publ. Dept. Math. Lyon 6 (2) (1969), pp. 75-84.

Regu par la Rédaction le 1. 6.1973 